\title{
Automated Cable Tracking in Sonar Imagery
}

\author{
Jason C. Isaacs and Ross Goroshin \\ Naval Surface Warfare Center \\ Panama City, FL USA \\ \{jason.c.isaacs1, rostislav.goroshin\}@navy.mil
}

\begin{abstract}
The classical paradigm of line and curve detection in images, as prescribed by the Hough transform, breaks down in cluttered and noisy imagery. In this paper we present an "upgraded" and ultimately more robust approach to line detection in images. The classical approach to line detection in imagery is low-pass filtering, followed by edge detection, followed by the application of the Hough transform. Peaks in the Hough transform correspond to straight line segments in the image. In our approach we replace low pass filtering by anisotropic diffusion; we replace edge detection by phase analysis of frequency components; and finally, lines corresponding to peaks in the Hough transform are statistically analyzed to reveal the most prominent and likely line segments (especially if the line thickness is known a priori) in the context of sampling distributions. The technique is demonstrated on real and synthetic aperture sonar (SAS) imagery.

Keywords - Edge detection, Hough transform, line detection, phase symmetry, anisotropic diffusion
\end{abstract}

\section{INTRODUCTION}

$\mathrm{T}$ HE inspection and maintenance planning of underwater sensor training ranges is considerably more cost effective and efficient if it can be performed by Autonomous Underwater Vehicles (AUVs). Therefore, the ability of an AUV to detect, track, and inspect the cable components of these ranges is of interest to the Navy.

Our initial goal is to detect prominent straight line segments. Lines in imagery vary in appearance, e.g., thickness, intensity (color), and geometry. It is necessary to formulate a general definition for cable-like objects which can be used to detect arbitrary cables in imagery collected by various sensors. We define cables as straight line segments, which have coherent structure, and whose intensity may be very close to that of the background (i.e., low SNR).

\section{A. Contributions}

This paper proposes an algorithm for the automated detection of cable-like objects in cluttered and noisy imagery. Most of techniques which are used in the algorithm are well known in the literature. Features used to classify the most statistically significant cable-like segments are introduced.

\section{B. Outline}

The remainder of this paper is as follows: Section II will provide some background information on the image processing techniques used in this work, review the mathematics behind these techniques, and identify the uniqueness of each. Section III will describe the methods used to analyze line segments and establish criteria for identifying cables and discuss the results. Section IV follows with a conclusion and a few thoughts on future work.

\section{IMAGE PROCESSING TECHNIQUES}

In this section we present an overview of the relevant image processing techniques used in the line detection algorithm. Most of these procedures are "off-the-shelf" techniques widely known/used by the image processing and computer vision communities. These are used to detect candidate line segments in the image which may or may not be portions of a cable. The last subsection proposes three measures, or features, which may be used to determine if a line segment is likely to be a cable.

\section{A. Anisotropic Diffusion}

Nonlinear anisotropic diffusion filters are iterative, "tunable" filters introduced by Perona and Malik [13], [14]. Gerig et al. used such filters to enhance MRI images [6]. Sapiro and Tannenbaum used a similar technique to perform edge preserving smoothing of MRI images [16]. In the extreme case, such smoothing might produce a profile of radio frequency inhomogeneity in the images. Others have shown that diffusion filters can be used to enhance and detect object edges within images [1], [13], and [14]. Perona and Malik formulated the anisotropic diffusion filter as a diffusion process that encourages intraregion smoothing while inhibiting interregion smoothing. Mathematically, the process is defined as follows:

$$
\frac{\partial}{\partial t} I(\bar{x}, t)=\nabla \cdot(c((\bar{x}, t) \nabla I(\bar{x}, t)))
$$

In our case, $I(\bar{x}, t)$ is the image. $\bar{x}$ refers to the image axes and $t$ refers to the iteration step. $c(\bar{x}, t)$ is called the diffusion function and is a monotonically decreasing function of the image gradient magnitude:

$$
c(\bar{x}, t)=f(|\nabla I(\bar{x}, t)|)
$$

It allows for locally adaptive diffusion strengths; edges are selectively smoothed or enhanced based on the value of the diffusion function. Although any monotonically decreasing continuous function of $\nabla I$ would suffice as a 
diffusion function, two functions have been suggested:

$$
\begin{aligned}
& c(\bar{x}, t)=\exp \left(-(|\nabla I(\bar{x}, t)| / K)^{2}\right) \\
& c(\bar{x}, t)=\left(1+\left(\frac{|\nabla I(\bar{x}, t)|}{K}\right)^{1+\alpha}\right)^{-1}, \alpha>0
\end{aligned}
$$

where $K$ is referred to as the diffusion constant or the flow constant. An example of the anisotropic diffusion technique of Equation (4) for noise removal is shown in right image of Figure 1 below for the original image, left.

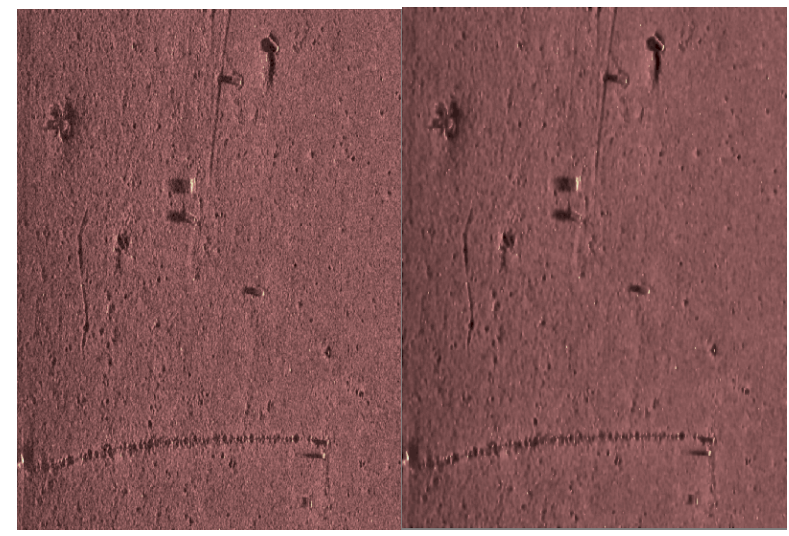

Fig. 1. Left, original sonar image showing speckled noise. Right, the result of completing ten iterations of equation (4).

\section{B. The Detection of Edges and Lines}

Edge detection refers to algorithms which aim at identifying points in a digital image at which the image brightness changes sharply, or more formally has discontinuities. Images contain step edges, line features and many feature types that are somewhere between the two. There are many methods for edge detection, but most of them can be grouped into two categories, search-based and zero-crossing based. The search-based methods detect edges by first computing a measure of edge strength, usually a first-order derivative expression such as the gradient magnitude, and then searching for local directional maxima of the gradient magnitude using a computed estimate of the local orientation of the edge, usually the gradient direction. The zero-crossing based methods search for zero crossings in a second-order derivative expression computed from the image in order to find edges, usually the zero-crossings of the Laplacian or the zero-crossings of a non-linear differential expression, as will be described in the section on differential edge detection following below.

As a pre-processing step to edge detection, a smoothing stage, typically Gaussian smoothing, is almost always applied as here we use the anisotropic diffusion technique discussed above. Edge detection methods that have been published mainly differ in the types of smoothing filters that are applied and the way the measures of edge strength are computed. As many edge detection methods rely on the computation of image gradients, they also differ in the types of filters used for computing gradient estimates in the $\mathrm{x}$ - and y-directions.

Traditional gradient based edge operators are tuned to detect step edges, and hence are unable to properly detect and localize other feature types. More adaptive non-gradient based techniques show certain promise in the areas of edge and line detection for Sonar based imagery.

\section{1) Phase Symmetry and Congruency}

The Phase Congruency [8], [9] detector is used as a tool to identify the different feature types found in images. It was noted that there is a continuum of feature types between step edges and ramps, and that most images have a broad distribution of all these feature types. It is concluded that in typical images gradient based operators detect and localize only a small fraction of features correctly.

In general the edge detection literature has concentrated on the detection of step edges. The principal criterion is usually the good detection and localization of step features in the presence of noise. This is typified by the work of Sobel [15], Marr and Hildreth [10], Canny [4], and many others. A very limited amount of work has been done on the detection of other kinds of features. Some exceptions to this are the line detection work of Canny [3], the detection of peaks and roofs by Perona and Malik [13], [14], the detection of steps and bars by Wang and Jenkin [19], and the catalog of feature types developed by Aw, Owens and Ross [2]. The emphasis on the detection of step edges is misplaced. Images contain a wide variety of edge types, many of which are somewhere between a step and a ramp. This method shows that one can describe a continuum of feature types between step edges and lines, and that most images have a broad distribution of all these feature types. The emphasis of computer vision research on the detection of step edges has resulted in edge detectors that fail to find, and/or incorrectly localize, valid features that are recognized by the human eye.

One approach to detecting relevant image features is to detect symmetry and asymmetry in image phase. The phase of the image along a given axis is computed by considering the transition of gray scale values along that axis. Points of symmetry and asymmetry correspond to salient image features such as lines (steps) and edges. Points of symmetry may be detected in the frequency domain as points where the frequency components attain a minimum or maximum. Similarly points of asymmetry may be detected in the frequency domain as points where the frequency components contain an inflection point. An example of the Phase Symmetry edge detection operation is shown for a sonar image in Figure 2. 

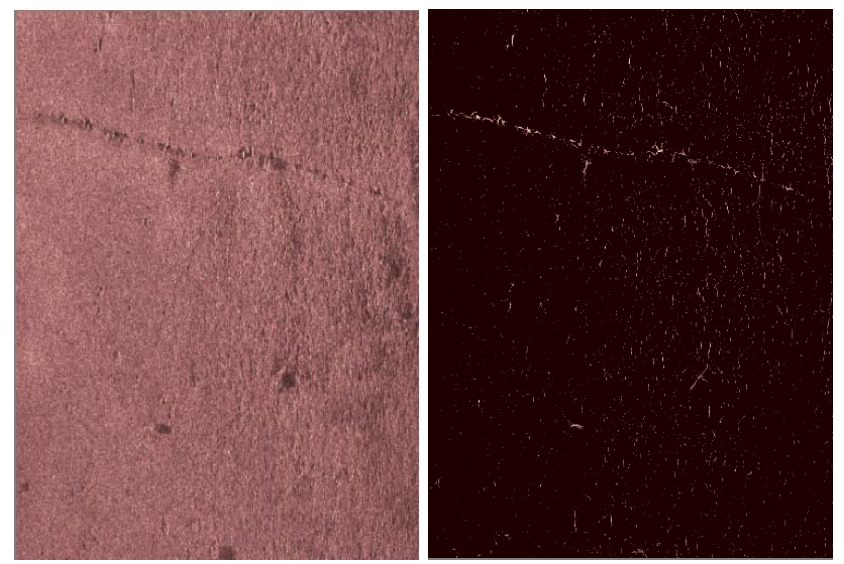

Fig. 2. An example of the Phase Symmetry edge detection operation for a SAS Image. The original image is on the left with the Phase Symmetry result on the right.

\section{2) Hough Transform}

In automated analysis of digital images, a problem that often arises is the detecting of simple shapes, such as straight lines, circles or ellipses. In many cases an edge detector can be used as a pre-processing stage to obtain image points or image pixels that are on the desired curve in the image space. Due to imperfections in either the image data or the edge detector, however, there may be missing points or pixels on the desired curves as well as spatial deviations between the ideal line/circle/ellipse and the noisy edge points as they are obtained from the edge detector. For these reasons, it is often non-trivial to group the extracted edge features to an appropriate set of lines, circles or ellipses. The purpose of the Hough transform is to address this problem by making it possible to perform groupings of edge points into object candidates by performing an explicit voting procedure over a set of parameterized image objects [17].

The simplest case of Hough transform is the linear transform for detecting straight lines. In the image space, the straight line can be described as $y=m x+b$ and can be graphically plotted for each pair of image points $(x, y)$. In the Hough transform, a main idea is to consider the characteristics of the straight line not as image points $\mathrm{x}$ or $\mathrm{y}$, but in terms of its parameters, here the slope parameter $\mathrm{m}$ and the intercept parameter b. Based on that fact, the straight line $y=m x+b$ can be represented as a point $(m, b)$ in the parameter space. However, one faces the problem that vertical lines give rise to unbounded values of the parameters $m$ and $b$. For computational reasons, it is therefore better to parameterize the lines in the Hough transform with two other parameters, commonly referred to as $r$ and $\theta$ (theta). The parameter $r$ represents the distance between the line and the origin, while $\theta$ is the angle of the vector from the origin to this closest point. Using this parameterization, the equation of the line can be written as

$$
y=\left(-\frac{\cos \theta}{\sin \theta}\right) x+\left(\frac{r}{\sin \theta}\right) .
$$

which can be rearranged to $r=x \cos \theta+y \sin \theta$ [17]. It is therefore possible to associate with each line of the image, a pair $(r, \theta)$ which is unique if $\theta \in[0, \pi]$ and $r \in \mathfrak{R}$, or if $\theta \in[0,2 \pi]$ and $r \geq 0$. The $(r, \theta)$ plane is sometimes referred to as Hough space for the set of straight lines in two dimensions. This representation makes the Hough transform conceptually very close to the two-dimensional Radon transform, whereby an infinite number of lines can pass through a single point of the plane. If that point has coordinates $\left(x_{0}, y_{0}\right)$ in the image plane, all the lines that go through it obey the following equation:

$$
r(\theta)=x_{0} \cos \theta+y_{0} \sin \theta
$$

This corresponds to a sinusoidal curve in the $(r, \theta)$ plane, which is unique to that point. If the curves corresponding to two points are superimposed, the location (in the Hough space) where they cross correspond to lines (in the original image space) that pass through both points. More generally, a set of points that form a straight line will produce sinusoids which cross at the parameters for that line. Thus, the problem of detecting collinear points can be converted to the problem of finding concurrent curves. The linear Hough transform can be simply visualized as the integration of image values along all possible lines which fit in the image, i.e., all possible combinations of $r$ and $\theta$.

Given the image of Figure 3, the series of peaks and values in the Hough transform image of Figure 4 correspond to the summations along the high (bright return) and low (shadow) of ripples which are clustered at about $45^{\circ}$ from the horizontal, or $180-45=135^{\circ}$ using our angle convention. Similarly, the cable in at about $85^{\circ}$ from the horizontal, which produces a peak at about $180-85=95^{\circ}$. Therefore long, straight, bright lines produce peaks in the Hough domain, while long, straight and dim lines (such as shadows) produce valleys. It is worth noting that had the cable run parallel to the ripples it would not be discernable as a separate peak in the transform domain.

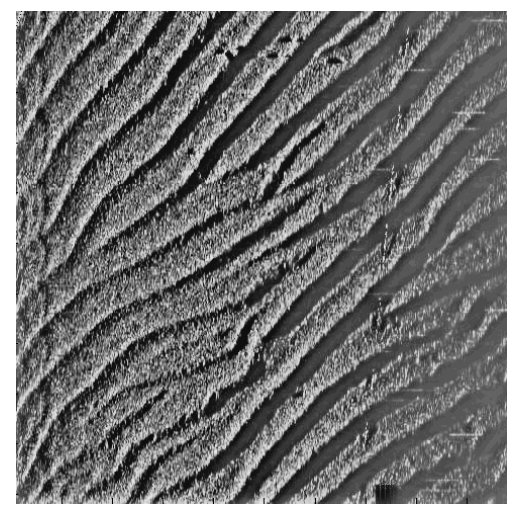

Fig. 3. Sonar Image with sand ripples. 


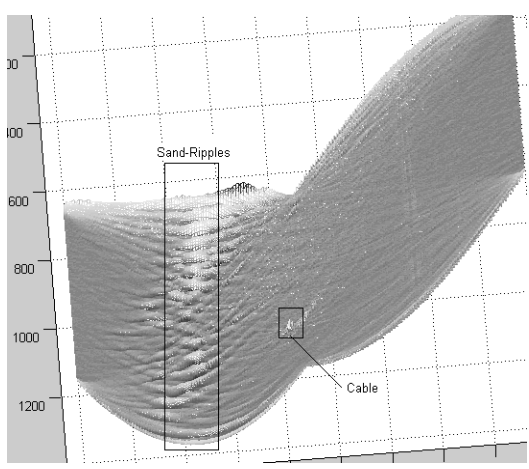

Fig 4. Hough Transform of the image of Figure 3.

\section{Line Segment Analysis}

Peaks in the Hough transform of the phase symmetry image provide a strong indication for the presence of straight line segments. However it is not generally guaranteed that the top peak of the Hough transform corresponds to the cable. Other linear objects such as anchor drag marks, bottom texture, or even spurious objects, coincidentally, forming a line will produce peaks in the Hough transform. Therefore, further processing and analysis is required to determine if a given peak in the Hough transform does, in fact, correspond to a straight cable segment.

In this section three measures are demonstrated for selecting cables from the top peaks in the Hough transform. Figure 5 (top left) shows the image of a highly textured background containing cable-like and other spurious objects. Figure 5 (top right) shows the corresponding phase symmetry transformed image. Computing the Hough transform of the phase symmetry image and plotting the line corresponding to the top peak in the transform (measure \#1) produces the blue line in Figure 5 (bottom). The red line in the same image corresponds to the densest line, exceeding some minimum length (measure \#2), of the top twenty peaks from the Hough transform. However, neither of these lines corresponds to the cable. The measure which produced the green line segment (measure \#3), which does indeed correspond to the cable is computed as follows:

1. Find the top n-peaks in the Hough transform

2. Determine the most dense line segments (exceeding some minimum length) corresponding to each peak

3. Compute the ratio of the mean intensity of the line segment to the mean intensity of its immediate neighborhood (see Figure 6)

4. Choose the line segment which corresponds to the highest such ratio

The green line segment in Figure 5 above exactly corresponds to the red rectangle in Figure 6 which produced the highest ratio measure mentioned above.

Intuitively, the ratio measure is an attempt to discard coincidental line structures. This measure can be justified in the context of sampling distributions. That is, if the phase symmetry image is assumed to be a two-dimensional random field, with mean $\mu_{\mathrm{f}}$ and variance $\sigma^{2}$, and a sample of it is taken along a line.

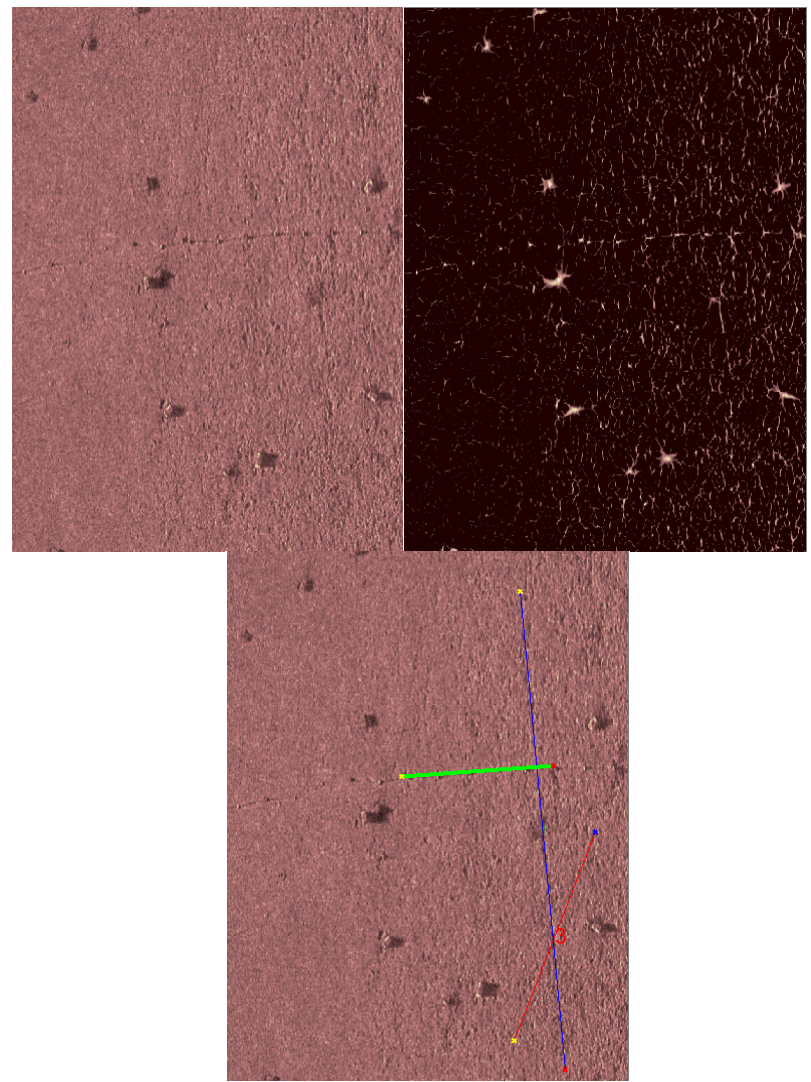

Fig. 5. Example of the cable detection results for measures one (blue line), two (red line), and three (green line).

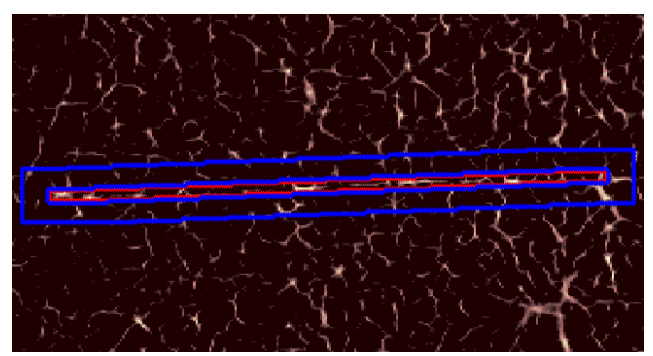

Fig. 6. A cable-like line region of interest, the red box defines the target measure and the blue box defines the neighborhood

Then what is the likelihood that the sample mean value, $\mu_{\mathrm{s}}$, is much greater than $\mu_{\mathrm{f}}$ ? It turns out, by the central limit theorem, that the distribution of $\mu_{\mathrm{s}}$ is the normal distribution, regardless of the distribution of the random field itself [18].

The mean and variance of this normal distribution are $\mu_{\mathrm{f}}$ and $\sigma^{2} / n$, respectively, where $n$ is the sample size. Therefore, it is extremely unlikely that the mean of any line segment (of appreciable length) will be much greater than that of its immediate neighborhood, suggesting an unnatural, possibly manmade cable-like object. Figure 7 (bottom) shows the detection of a cable in a large, high resolution image. In this case, the densest line segment (measure \#2, red line) also produces the highest ratio value (measure \#3, green line). The top peak in the Hough transform (measure 
\#1, blue line) corresponds to a false detection.

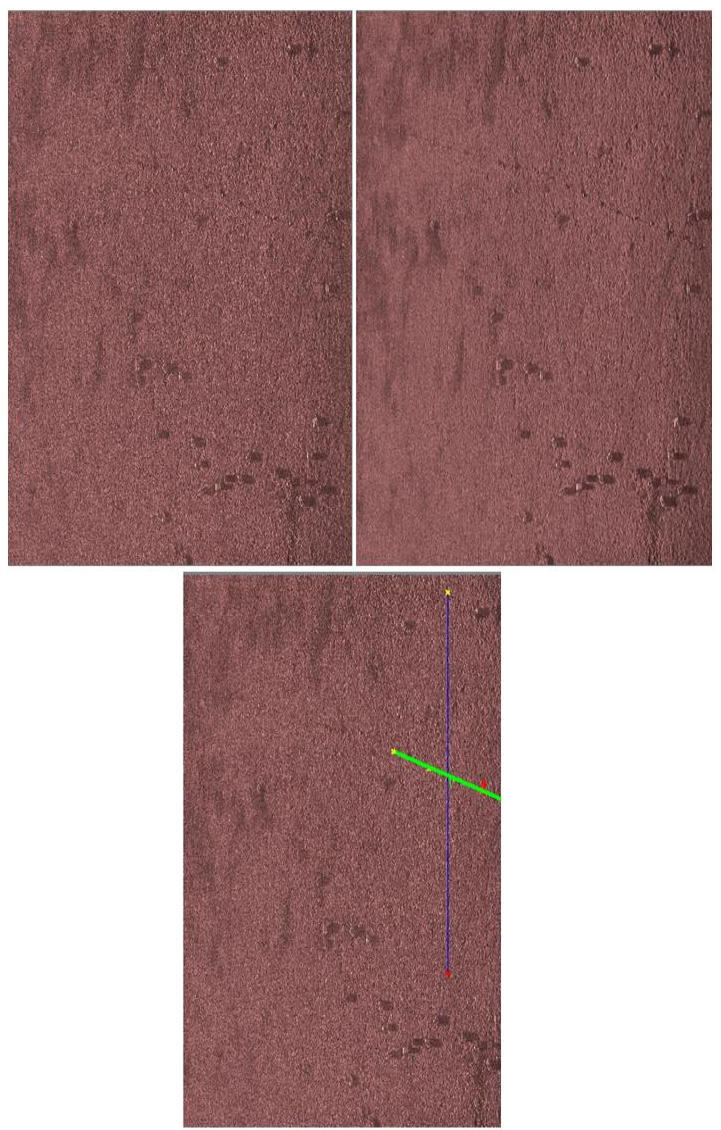

Fig. 7. Cable detection in a large high resolution image (Left: original, Right: de-noised, Bottom: detection).

Next, we examine an image which contains two cables of different thickness (see Figure 9). The right image shows that measures $\# 1$ and $\# 2$ both detect the longer, thinner cable, however measure \#3 results in the detection of the thicker, but less prominent cable. This is because the width of the rectangle (red rectangle in Figure 6 above) exactly matches the width of the cable detected by the green line, resulting in very high sample mean.

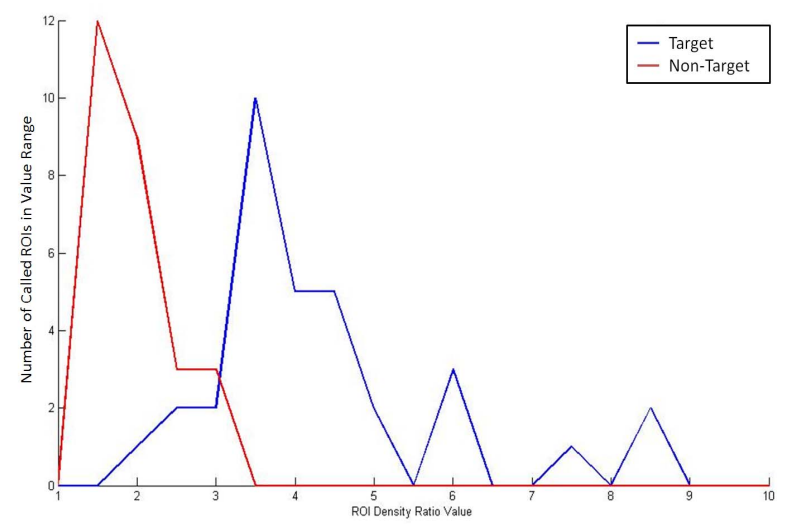

Fig. 8. Plot of the density ratio measure (measure \#3) distributions for cable and non-cable like regions of interest.

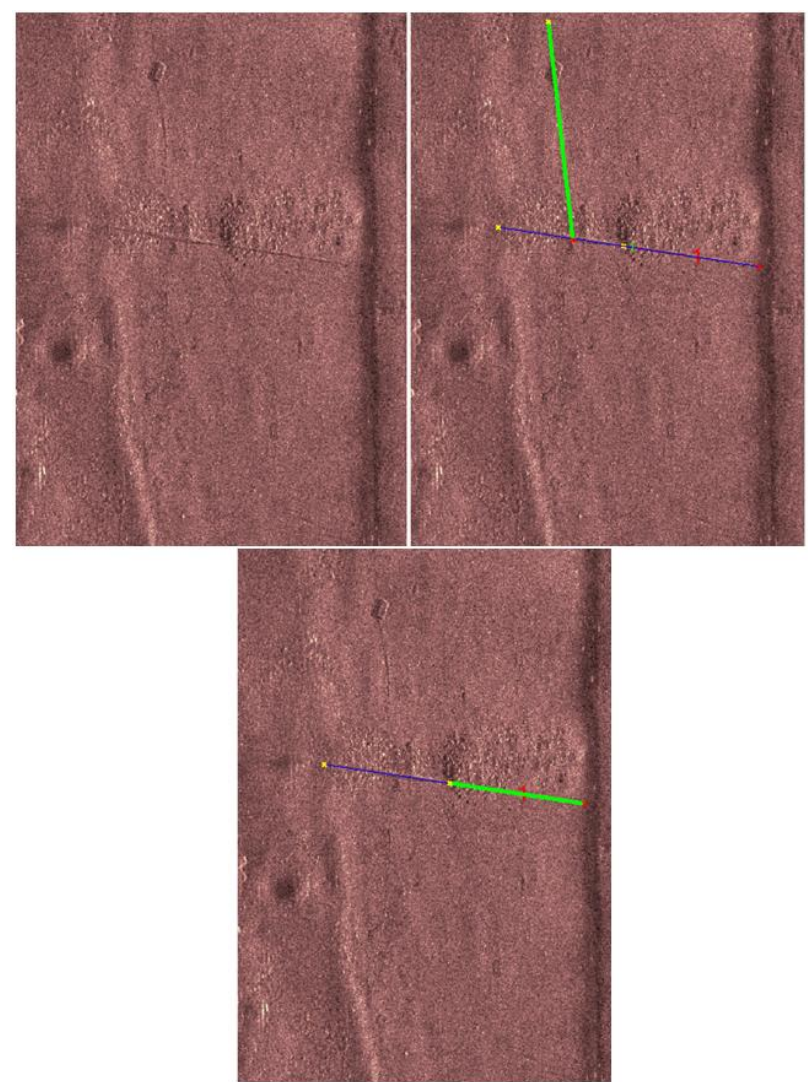

Fig. 9. Detection within a varied cable thickness image.

Upon decreasing the width of the inner rectangle measure \#3 agrees with measures $\# 1$ and $\# 2$, as seen in the bottom image of Figure 9. This suggests that if the expected cable width is known, more accurate detections are possible. The plot below (Figure 8) compares the density ratios for cable and not cable-like regions of interest calls.

Table I. Statistics for the cable call density ratios for the regions of interests in the imagery data.

\begin{tabular}{|c|c|c|}
\hline & Not Cable & Cable \\
\hline Mean & 1.92 & 4.37 \\
\hline Max & 3.12 & 8.75 \\
\hline Min & 1.27 & 1.98 \\
\hline
\end{tabular}

Table I lists the means, maximums, and minimums for the cable and non-cable like object calls. As shown, the cable density ratios are on average greater than non-cable ratios.

\section{REVIEW OF THE APPROACH}

Detection of straight line segments in images is considered a classical problem in image processing. A standard technique for detecting straight line segments involves the use of the linear Hough transform. However, due to the presence of spurious objects and textures common in seafloor imagery combined with very low SNR, straightforward application of the Hough transform does not yield reliable detection. Therefore lines in the image detected by the Hough transform are treated as "lines of interest" analogous to "objects of interest" in mine detecting $\mathrm{CAD} / \mathrm{CAC}$ algorithms. These lines of interest are further 
analyzed and classified as either cable or not cable based on their structure and the background statistics in their surrounding area.

The cable detection algorithm may be divided into five steps:

1. Noise Reduction

2.Edge and Line Enhancement

3. Thresholding

4.Line Detection

5.Classification

Figures 10 through 12 show the detection process. Figure 10 contains the original image and the noise-reduced image. The phase symmetry image, as shown in Figure 11 on the left, highlights coherent structures in the image. Candidate lines obtained via the Hough transform are shown in Figure 11 on the right, overlain on the image. Finally, three detected segments, using three different measures for classification are shown in Figure 12.

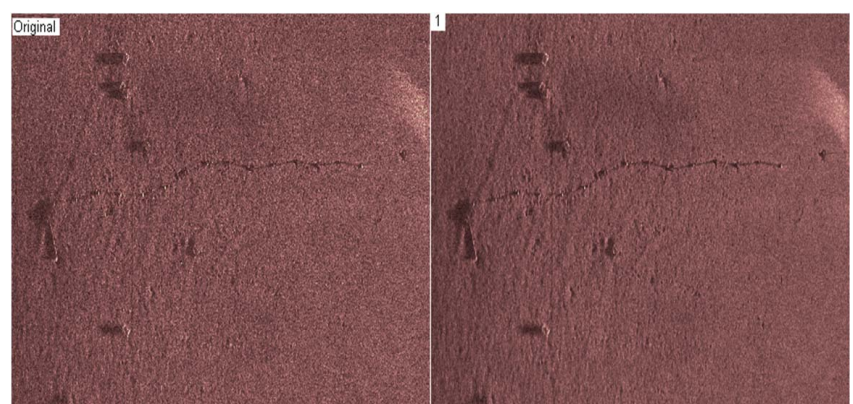

Fig. 10. The original image and step one of the cable detection procedure.

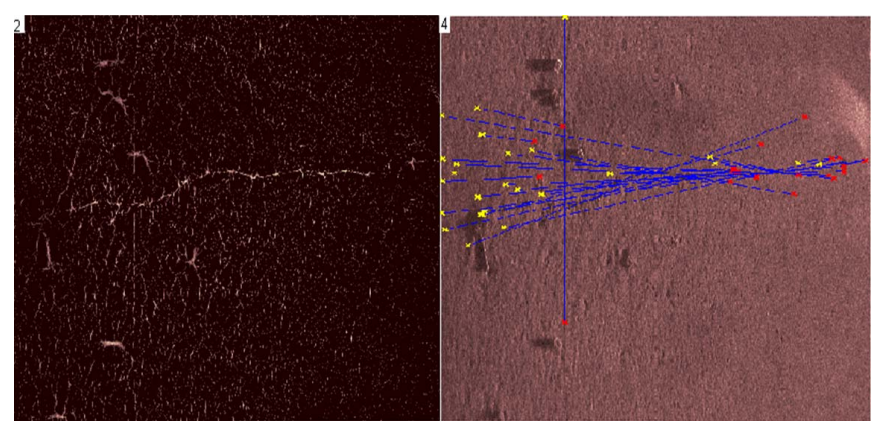

Fig. 11. Steps two and four of the cable detection procedure.

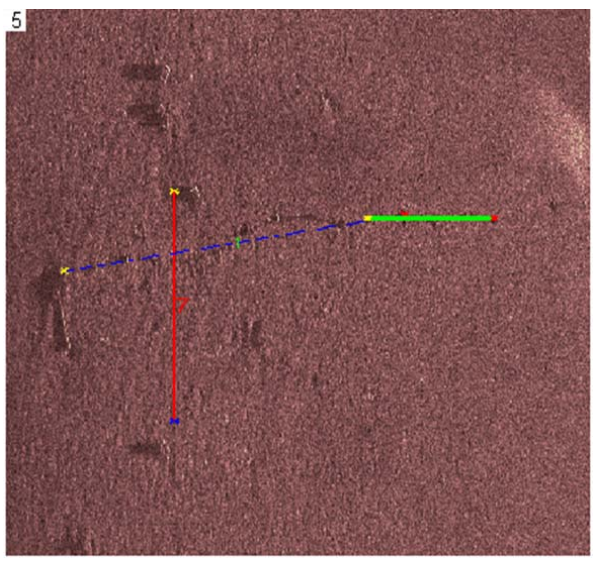

Fig. 12. Step five of the cable detection procedure.

\section{TRACKING CABLES}

After the initial detection of a cable in the first image of a sequence, future detections will focus on regions of interest about the location expected given previous heading and distance information in order to cut down on computation costs. However, if the cable cannot be found in subsequent image regions then the entire image will have to be searched in order to regain cable contact. Cable tracking through sonar image sequences is performed through edge-linking the previous cable of interest with the most cable-like-object in the next sonar image. Figure 13 shows a mosaic of images and the highlighted cable tracked through this sequence.

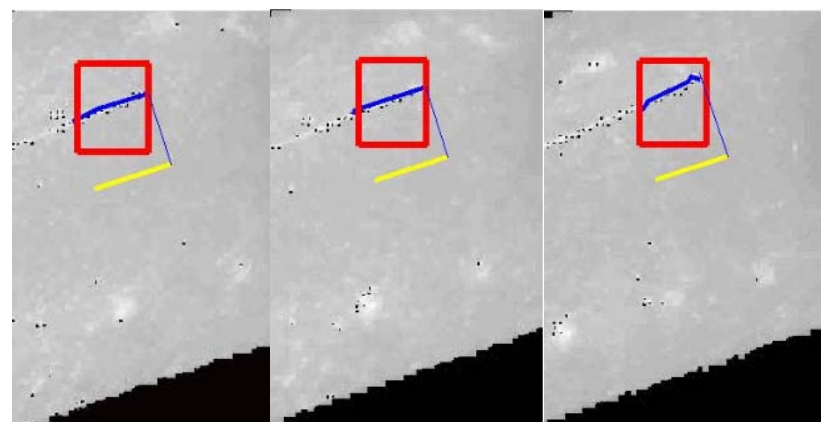

Fig. 13. Sequence of sonar images with a vehicle (yellow line) tracking a cable (blue line) along a run. The region of interest is the red box. The thin blue line represents a vector from the front of the vehicle to the end of the cable.

The results of tracking sequence in Figure 13 demonstrate that ability of our detection algorithm to capture the most cable like object in subsequent regions of highest probability of detection through a sequence of sonar images.

\section{CONCLUSIONS}

In this study we have presented an approach for detecting and tracking cable-like objects in imagery. Since this approach proved successful in cluttered imagery we believe that the same approach (or a simplified version of it) can be readily applied to most imagery where the cable exhibits some response. The approach was tested on available imagery which contained cables of opportunity. Other environments may present a greater challenge to our approach; therefore, future work should involve the use of imagery with the greatest combination of cable orientations and backgrounds.

\section{ACKNOWLEDGMENT}

This work was supported by US Naval Facilities Engineering Command.

\section{REFERENCES}

[1] Luis Alvarez, Pierre-Louis Lions, and Jean-Michel Morel. Image selective smoothing and edge detection by nonlinear diffusion. SIAM J. Number. Anal., 29(3):845-866, June 1992.

[2] Y K Aw, R Owens, and J Ross A catalogue of 1-D features in natural images, CVGIP - Graphical Models and Image Processing, (1994). 
[3] E.J. Candes and D.L. Donoho. Curvelets, A Surprisingly Effective Nonadaptive Representation For Objects with Edges. In: Saint-Malo Proceedings.

[4] J. F. Canny. A computational approach to edge detection. IEEE Trans. Pattern Analysis and Machine Intelligence, 8(6):112-131, 1986.

[5] Duda, R. O. and P. E. Hart, "Use of the Hough Transformation to Detect Lines and Curves in Pictures," Comm. ACM, Vol. 15, pp. 1115 (January, 1972)

[6] Guido Gerig, Olaf Kübler, Ron Kikinis, and Ferenc A. Jolesz. Nonlinear anisotropic filtering of MRI data. IEEE Transactions on Medical Imaging, 11(2):221-232, June 1992.

[7] P.V.C. Hough, Machine Analysis of Bubble Chamber Pictures, Proc. Int. Conf. High Energy Accelerators and Instrumentation, 1959.

[8] P. D. Kovesi. A dimensionless measure of edge significance from phase congruency calculated via wavelets. In First New Zealand Conf. on Image and Vision Comp., pages 87-94, Auckland, August 1993.

[9] P. D. Kovesi. Invariant Measures of Image Features From Phase Information. PhD thesis, The Univ. of W. Australia, May 1996.

[10] D. Marr and E. C. Hildreth. Theory of edge detection. Proceedings of the Royal Society, London B, 207:187-217, 1980.

[11] N. Nordström. Biased anisotropic diffusion - a unified generalization and diffusion approach to edge detection. Image Vision Comput., 8(4):318-327, 1990.

[12] R. A. Owens, S. Venkatesh, and J. Ross. Edge detection is a projection. Pattern Recognition Letters, 9:223-244, 1989.

[13] P. Perona and J. Malik. Detecting and localizing edges composed of steps, peaks and roofs. In Proceedings of 3rd International Conference on Computer Vision, pages 52-57, Osaka, 1990.

[14] Pietro Perona and Jitendra Malik. Scale-space and edge detection using anisotropic diffusion. IEEE Trans. on Pattern Analysis and Machine Intelligence, 12(7):629-639, July 1990.

[15] (Sobel) K. K. Pringle. Visual perception by a computer. In A. Grasselli (ed.), Automatic Interpretation and Classification of Images, pages 277-284. Academic Press, New York, 1969.

[16] Guillermo Sapiro and Allen Tannenbaum. Edge preserving geometric smoothing of MRI data. Tech. rep., Univ. of Minn., April 1994.

[17] Shapiro, Linda and Stockman, George. "Computer Vision," PrenticeHall, Inc. 2001

[18] Walpole, Myers, and Ye. Probability \& Statistics for Engineers and Scientists 7th Edition.

[19] Z. Wang and M. Jenkin. Using complex Gabor filters to detect and localize edges and bars. In Colin Archibald and Emil Petriu (eds.), Advances in Machine Vision: Strategies and Applications, World Scientific Series in Computer Science, volume 32, pages 151-170. Singapore, World Scientific Press,1992. 